International Journal of Biology, Pharmacy and Allied Seiences (IJBPAS)

'A Bridge Betueen caboratory and QRendo'

WwW.ijbas.com

\title{
BIG DATA FOR SMART APPLICATIONS: SMART APPLICATIONS, MAIN SOURCE OF COMMUNICATION BETWEEN THEM - IoT, BIG DATA
}

\author{
RAGIMOVA NAZILA A ${ }^{1}$, ABDULLAYEV VUGAR H ${ }^{*}$ AND ABUZAROVA \\ VUSALA A ${ }^{1}$
}

1: Department of Computer Engineering, Azerbaijan State Oil and Industry, University, Baku, Azerbaijan

*Corresponding Author: E Mail: Dr. Abdullayev Vugar H: abdulvugar@mail.ru Received $19^{\text {th }}$ July 2021; Revised $20^{\text {th }}$ Aug. 2021; Accepted $29^{\text {th }}$ Sept. 2021; Available online $1^{\text {st }}$ Nov. 2021 https://doi.org/10.31032/IJBPAS/2021/10.11.1004

\begin{abstract}
The development of artificial intelligence has led to an increase in the number of smart applications, smart devices, in short, "smart" technologies. These technologies can communicate with each other. The ability to make connections via IoT allows you to increase the number of connected devices. This leads to large data exchanges. It is good to have a lot of information, but a lot of information causes certain problems in their proper management. The large size of this information makes the problem even bigger. The article discusses the existence of Smart Technologies, IoT, which is the main source of communication between them, and Big Data technology, which is related to the analysis and management of large amounts of data. The combined use of IoT and Big Data has been reviewed, and the application of these two technologies in Smart Cities, the main application center, has been reviewed.
\end{abstract}

Keywords: Big Data, Smart Cities, Internet of Things, Smart Technologies, Smart Device

\section{INTRODUCTION}

It is known that the number of technologies In other words, such technologies allow based on artificial intelligence is growing. customers to "use" themselves, even if they 
do not have the ability to use the technology. In simpler terms, they free the user from knowing the main features of the technology in front of him. It is this component that makes them one of the most sought-after technologies by humans.

Such technologies can be called smart devices, smart applications, smart technologies. In fact, there are slight differences between these technologies. That is, these three terms cannot be equated.

Smart applications are implemented by IoT technology as basic means of communication between smart devices. Thereby, smart devices ensure the existence of İoT technology.

The interaction of smart devices with each other leads to redundant information. The abundance of information makes it difficult to manage them properly. To overcome this problem, experts worked on the development of another technology and there was used Big Data technology for proper data management.

\section{SMART APPLICATIONS}

Smart Applications are innovative systems that collect large amounts of data from sensors and other sources, using machine learning algorithms and predictive analytics to make this or that information more effectively for users and improve their experience. (1) [8].
The main distinguishing features of smart applications are:

\section{They are $S M A R T$}

Smart apps use analytics, machine learning, and artificial intelligence services to give users and items tips and predictions to take the next best steps.

\section{They are CONTEXTUAL}

Using personal, sensor, and location information, smart applications are personalized, incorporated into user processes, and available on any channel/device.

\section{They are $A C T I V E$}

Smart apps use notifications, chatbots, and messaging services to actively interact with users and give them smart advice on what to do.

Smart devices, including artificial intelligence and machine learning, are all everyday objects that have been intelligentized with advanced computing and networked to create the Internet of Things (IoT).

Smart devices can be integrated to bring intelligence to both objects and spaces, such as smart homes and buildings, and can help automate processes and controls. They can be used in almost every industry, from smart manufacturing to healthcare, helping to increase efficiency and optimize operations. 
The definition of smart devices is as follows: [5] "A smart device is a contextaware electronic device capable of autonomous computing for communication between other devices and the ability to wirelessly connect to them." (2)

The three main features of smart devices are given in this definition:

\section{$>$ Be aware of the context \\ $>$ Autonomous calculation $>$ Contact}

This definition is consistent with the basic idea of IoT, that is, any "thing" can be part of IT. If we add a sensor, small computing power, and a network connection, a simple device can turn into such a smart device.

We mentioned earlier that smart devices and smart applications are not synonymous. But there are similarities. This can be seen from the definitions given to them.

The following expression can be taken as a common conclusion from the definitions given for both:

\section{Data exchange}

It should be noted that the basis of both technologies is the correct exchange of data.

In fact, the main goal of many technologies is to exchange information properly. In order to exchange accurate information, these technological devices must interact with each other.

For example, let's take a simple example: Suppose you have a smart refrigerator in your home. And it's aware of all the food placed inside that refrigerator. This means that your device itself will alert you to which foods in the refrigerator are running out. When the eggs, milk, etc. in a refrigerator run out, the refrigerator will send a warning signal to the device connected to it. This connected device can be a phone, computer, tablet, or any similar device.

This connection is made using IoT. These devices connect to each other to provide an Internet of Things network.

MAIN COMMUNICATION TECHNOLOGY - INTERNET OF THINGS

The Internet of Things is a collection of devices that interact with each other. Which means that these devices are "smart" in themselves. The main fundament of the Internet of Things technology is the interconnection of devices. That is, it is smart devices and their connection to each other. Smart devices here are usually connected to other devices or networks, Bluetooth, Zigbee, NFC, Wi-Fi, LiFi, 5G, etc. includes electronic devices that are connected via certain wireless protocols, 
such as, to some extent, interactively and autonomously [6].

Types of smart devices are smartphones, smart vehicles, smart thermostats, smart doorbells, smart locks, smart refrigerators, and so on.

IoT is a complex system with several features. These features are:

1) Intelligence means the intelligence of devices. Of course, this intelligence is artificial intelligence. Intelligent devices based on artificial intelligence. Naturally, the devices communicate with each other due to these features.

2) Connection is the connection between smart devices. These connections are a key component of the IoT network.

3) Dynamic nature is synonymous with the Internet of Things ecosystem. Which is also the IoT network.

4) Huge scale. With each passing day, the number of connected devices, which are a key component of the Internet of Things ecosystem, is constantly increasing. According to statistics, by 2025 the number of IoT devices will reach 100 billion.
5) Data. The interconnection of devices allows the exchange of information. "The right information can help to make the right decision". Interconnected devices can provide the user with the necessary information at the right time. For example, the simple refrigeration issue mentioned above.

6) Perception. This is mainly done through sensors. Through sensors, devices can perceive the environment, receive, and warn accordingly.

7) Security. One of the main characteristics of IoT devices is the implementation of security. There can be a security vulnerability in any technology. In addition, one of the main problems that professionals have to solve is security. Devices need to operate in a secure ecosystem for proper data exchange.

If any device can connect to the Internet and has sensors that transmit data, this device can be considered an IoT device.

The Internet of Things also includes an extensive network of large-scale data, all 
of which are integrated into the everexpanding IoT ecosystem.

All these common devices are called smart IoT devices. IoT devices are mainly smart devices that support internet connection, interact with other devices on the internet, and allow a user to access it remotely to manage the device according to their needs.

IoT devices include sensors that work on data collected by sensors through machine learning and mini-computer processors. In fact, IoT devices are minicomputers connected to the Internet and are vulnerable to malware and hacker attacks.
Machine learning is the study of computers in a human-like way - by gathering information from the environment, and this is what makes IoT devices smart. This information can help the machine learn your options and adjust itself accordingly. Machine learning is a type of artificial intelligence that helps computers learn without programmed by anyone.

Smart cities are the main space for smart devices, IoT devices, and IoT networks. The concept of smart cities is illustrated in Figure 1:

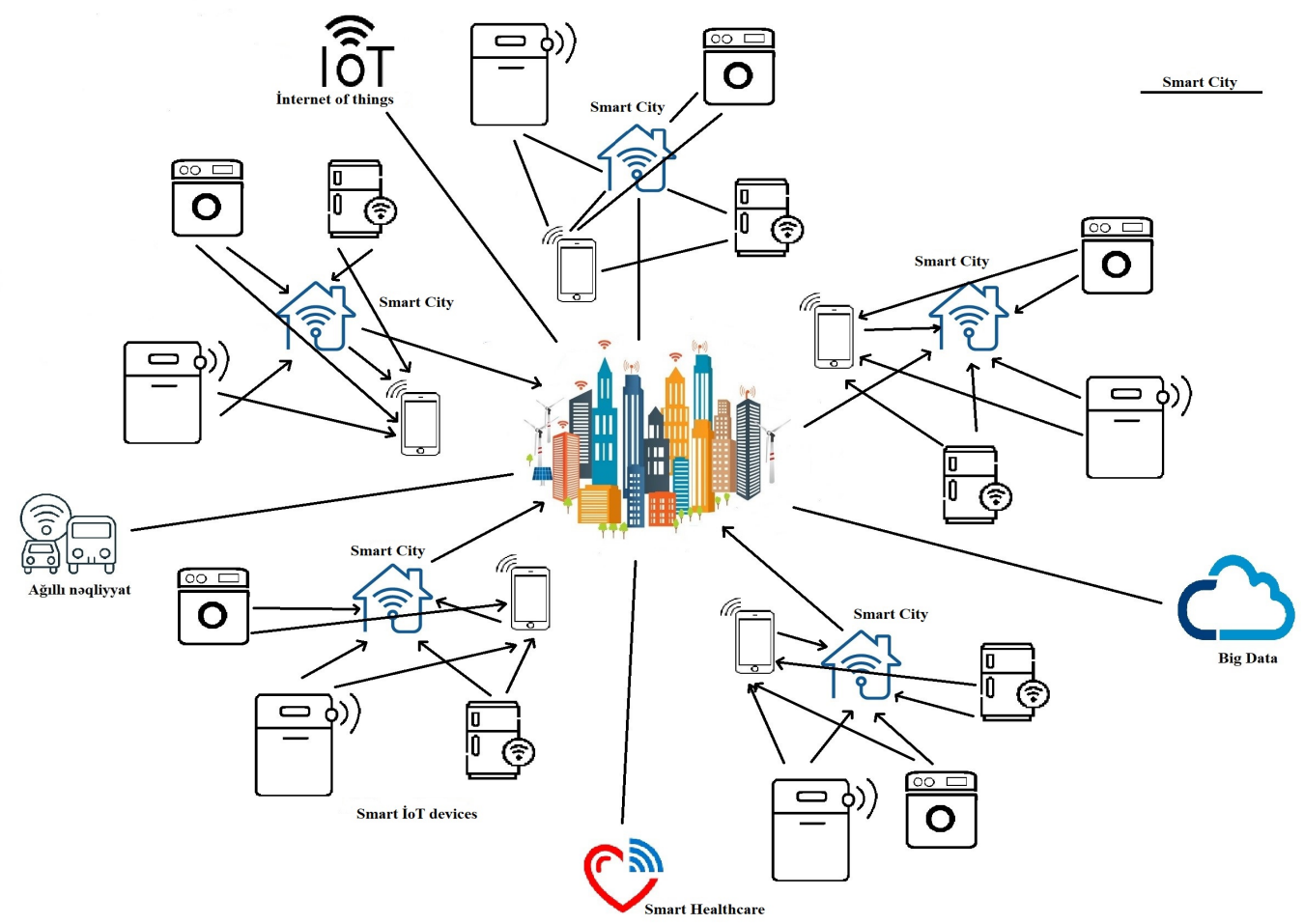

Figure 1: Smart City as a space for basic smart IoT devices

We can say that smart cities are the means that any IoT device can be found main place of the Internet of Things. This within Smart Cities. 
The main process between devices is the exchange of information. This leads to redundant information as a result. This information is obtained correctly and is used in decision-making.

In this regard, access to data, especially how large amounts of data can be obtained, stored, protected, processed, integrated into decision-making mechanisms, etc., has recently led to the development and use of big data technology.

\section{WHAT IS BIG DATA}

John Mashey, a computer scientist at Silicon Graphics, first used the term big data in 1998.

The information offers great benefits and opportunities for organizations today. At the World Economic Forum in Davos in 2012, in addition to values such as money and gold, "information" was marked as a new economic value.

It is very difficult to define Big Data accurately because the concept of "big" varies from one area to another in terms of the amount of data. It is not defined by a set of technologies, but by a category of techniques and technologies [21]. In this regard, it is possible to give different definitions of Big Data.

The main features of Big Data are known as $5 \mathrm{~V}$, and they are:
1. Volume represents the amount of data created, stored, and processed in the system. The amount of information is very important. This can be seen in the name of technology. The increase in volume is explained by the need to use it at the same time as the amount of information created and stored.

2. Velocity represents the frequency at which data is created, captured, and shared. Data comes in streams and must be analyzed in real-time.

3. Variety. When data is obtained, they come in a variety of unstructured forms. Audio, video, picture, text, etc. It represents the multiplication of data types managed by an information system.

4. Veracity refers to the accuracy and precision of a particular set of data. This includes evaluating the source of the information - whether it is reliable or not.

5. Value is The most important among $5 \mathrm{~V}$. However, the fact that this information has no value in realtime is not good for the user. In this regard, this component is the most "valuable" among 5V. 


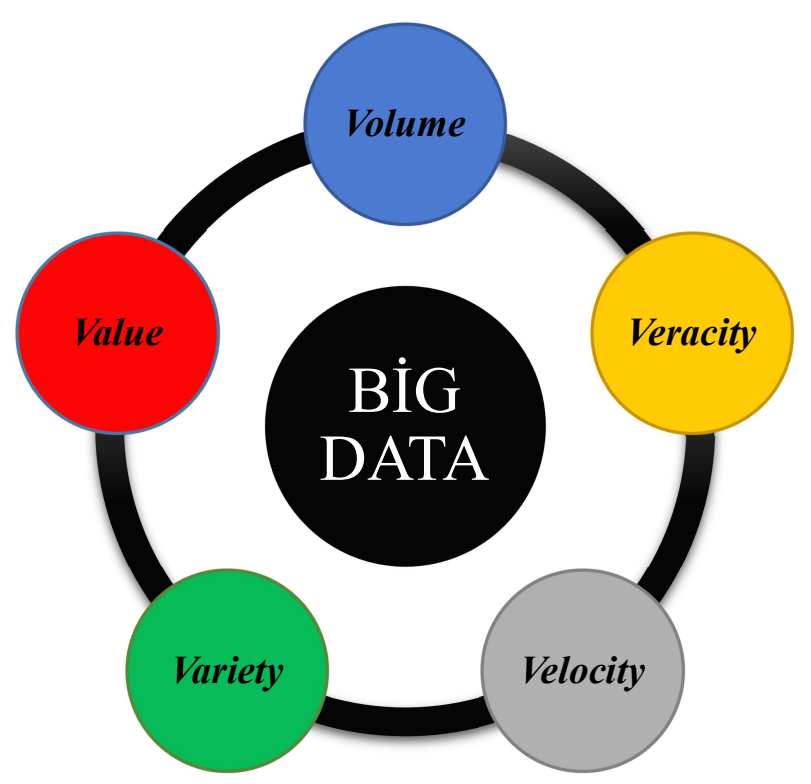

Figure 2: $5 \mathrm{~V}$ of Big Data

Simply put, Big Data is the product of the attention paid to information that must be protected and not lost as a result of the latest technological developments.

\section{BIG DATA AND IoT}

Given that data exchange is central to IoT, it is also possible that there is a link between IoT and Big Data.

Big Data and tools created for analysts are useful for correcting the flow of data flowing from IoT devices. IoTcentric developers create platforms, software, and applications that businesses and organizations can use to manage IoT devices and generated data.

Big Data and IoT are different ideas, but they depend on each other to be extremely successful. Both emphasize the importance of transforming information into tangible material concepts.
An example of IoT working with Big Data analysts comes from the transportation sector. Shipping companies connect IoT sensors to trucks, planes, boats, and trains to monitor speed, stopping, engine status, and other information. They can use this information to make immediate decisions and wait for repairs to be made in the near future, while also storing the information gathered to see the company's development over time. As a result, this combination of immediate IoT insights and long-term Big Data analytics results in cost savings, increased efficiency, and better use of environmental resources [7].

IoT and Big Data have an important connection that will continue to evolve as technology advances. Companies that want to take advantage of the power of data need to carefully consider the devices they 
choose to deploy and the types of data they collect.

In fact, IoT involves sensors placed on any type of device and sending data streams to one or more central (Cloud) space via Internet streams. This information can be analyzed later. These results are used to improve the user's life. All IoT devices follow these five basic steps: measuring, sending, storing, analyzing, and moving.

It is necessary to increase the speed and accuracy of Big Data analysis to ensure the implementation of IoT solutions. However, it should be noted that Big Data is not completely useful here. The potential

of Big Data can only be realized when combined with artificial intelligence.

In general, it should be noted that Big Dara and IoT, no matter how common they are, are completely different concepts. Both can exist independently of each other.

Here are a few examples of how Big Data and IoT are used together:

\section{1) DİSNEY - DİSNEY} MagicBand

Disney MagicBand is a special bracelet and collects all kinds of information from visitors. As a result, it is used for the visitors themselves. This information helps to enter parks, unlock hotel rooms and buy food, etc. can be used [13].

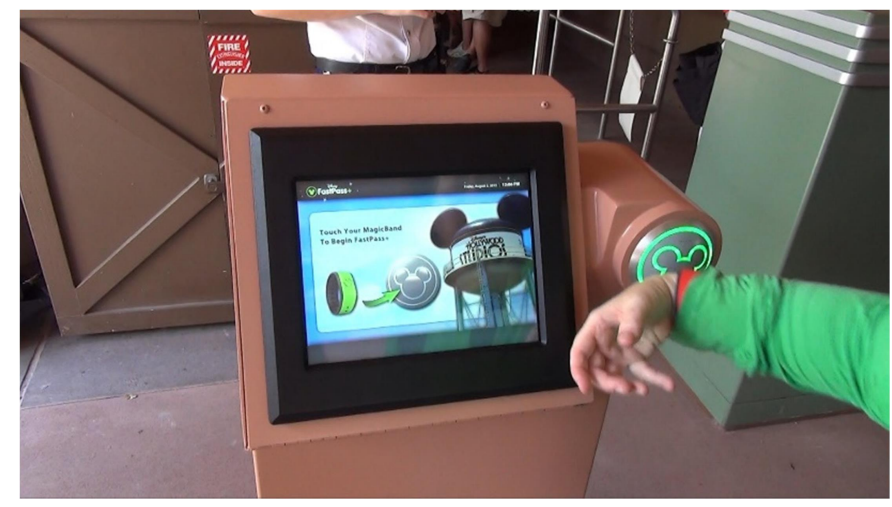

Figure 3. Disney MagicBand and sensors

For this, special sensors located around the park are used. They receive information about visitors with sensors. They communicate with thousands of sensors around the park and transmit real-time data for analysis [10].
In addition, it also reduces fraud and speeds up the experience inside the park by eliminating queues.

2) UPS - UNITED PARALEL SERVİCE

UPS is an American multinational package delivery and supply chain management company. Drivers use 
Big Data analytics to determine the optimal delivery route.

UPS has equipped the mass delivery fleet with sensors that record about 200 data points each. The result is lower fuel consumption (and this saves money) and reduced impact on the environment through less waste.

According to a recent Forbes report, UPS's On-Road Integrated Optimization and Navigation (ORION) "uses advanced algorithms to create optimal routes for drivers from data transmitted by customers, drivers, and vehicles; and can change routes immediately based on changing weather conditions or accidents. "

\section{3) TempuTech}

TempuTech is another IoT and Big Data system that affects the agricultural industry. The company offers closed systems that track optimal grain storage and potential hazards in systems such as grain elevators.

The collected data is transmitted to farm operators and used to predict changes in air and humidity and temperature.

4) John Deere
Another example of Big Data and IoT's use in agriculture is equipment manufacturer John Deere.

John Deere offers several effective information services that allow farmers to benefit from realtime monitoring of data collected from users of John Deere equipment. This information allows farmers to make better decisions that will help them manage their farms and machines more intelligently. Environmental sensors also measure "air and soil temperature, wind speed, humidity, solar radiation."

This information will help farmers find that their crops have reached optimal moisture levels. Armed with this information, farmers can make timely irrigation decisions.

The online portal allows farmers to access data collected from sensors connected to their machines while working in the fields, as well as data collected from other users around the world [13].

Another information-based service is the John Deere Operations Center, which helps farmers decide which plants to plant-based on the information they 
collect in their fields and in the products of other users.

5) BC HYDRO

BC Hydro, located in the Canadian province of Central British Columbia, is an electricity company that supplies power to about 2 million Canadians. In 2011, the company began converting electricity meters to smart meters [13].

Users can now track their energy usage by the hour and see trends in their usage data. This has significantly reduced electricity theft. At the same time, the company is notified when automatic power outages are detected in a certain area. Using the data collected, BC Hydro can provide advanced service at a lower cost.

There are quite a few examples of Big Data. We have looked at some of the examples above.

\section{BIG DATA AND SMART CITIES}

One of the major central ecosystems of smart technology is Smart Cities. Because there are not only smart devices, smart applications, but also smart connections. In this regard, Smart Cities is also home to technologies such as Smart Home, IoT, and Big Data. It is relatively interesting that these technologies are available in larger areas instead of IoT or Big data within a small company.

Both IoT and Big Data have applications in Smart Cities. As mentioned earlier, these two areas are different from each other, but their use in the field of application is very wide. The above examples were also cases where IoT and Big Data were used together. However, these cases can occur in any place.

This chapter will look at Big Data applications in Smart Cities.

Smart cities are taking advantage of technological innovations to improve important aspects of our quality of life.

The main goals for any Smart City are as follows: [14]

$\checkmark$ Improving the quality of life of citizens

$\checkmark$ To develop the knowledge economy

$\checkmark$ Create a living ecosystem

Smart Cities is a place where technology comes to life. It can be said that the main flow of life here is the integration of technology. Smart government, smart economy, smart transportation, smart health, and finally smart people.

In general, the main technological innovations used in Smart Cities are: [14] Internet of Things Big Data Cloud computing 
Geographic Information Systems

\section{Green technology}

Mobile technology

These technologies are the key tools to help keep the Smart City afloat.

The key to a smart urban ecosystem is that smart devices are properly connected to each other. That is one of the key components designed to improve quality of life. For example, the presence of smart traffic lights to control the flow of traffic significantly reduces the percentage of accidents. Or smart fire control systems installed in smart homes will alert the fire brigade in the event of a fire, even if you are not at home. The main goal of a smart city, as mentioned above, is to improve the quality of life as intended.

All these connections are made through IoT. However, this process is not limited to IoT. If the IoT devices are connected properly and the information obtained is not managed properly, this can prevent the process from working well.

Data management refers to how data is obtained, stored, protected, processed, and integrated into decisionmaking and learning mechanisms. It is especially important to manage large amounts of data. This is one of the main goals of Big Data. That is, these opportunities are provided to users through Big Data.

In fact, the above-mentioned operations, ie data acquisition, storage, protection, etc. no matter how complicated it may seem, are processes that can happen in a matter of seconds with the right technology. It is worthwhile to give a simple example accordingly.

Suppose a thief breaks into your home while you are out of town. However, because your home is a smart home within a smart city, indoor surveillance systems can protect you from this unwanted event. But for that, IoT and Big Data must work together.

A person who enters the house is considered a thief if he does not meet the requirements of the system. In this case, the control system correctly analyzes and transmits information about the theft to the police team. We can show the sequence of processes as follows:

1) The system obtains the necessary information from the person entering the house. That is, the system analyzes it.

2) The obtained data is immediately compared with the data in the system's internal database.

3) After accepting that the person being compared is not the owner 
of the house, this information is stored and a warning signal is sent directly to the police.

That is, at this point, the system makes a decision on its own and sends a warning signal using the information correctly.

Although the sequence is longer in detail, all these processes take place within a few minutes, depending on the system.

However, it should be noted that these technologies themselves are based on artificial intelligence, which can occur in cases such as erroneous analysis, even if not high. That is, small mistakes are inevitable. If the person entering the house is you, the system may perceive you as a thief.

\section{BİG DATA APPLİCATION İN SMART CITIES}

The application of Big Data technologies for smart cities allows efficient data storage and processing to produce data that can develop different smart city services [23]. There are various areas where Big Data applies. These are:

1. Smart Grid

2. Smart Transportation

3. Smart Governance

4. Smart Healthcare

5. Smart Energy Management

6. Smart Security

7. Smart Education

\section{Smart Grid}

The rapid deployment of smart grids has allowed researchers to combine, analyze and use real-time energy production and consumption data and other environmental data [15].

Numerous data analytics algorithms and applications aimed at data analysis are applied to the smart network. The main job of Big Data in a smart network is to extract valuable information from the data obtained and plan future activities and operations.

Smart network data analytics can also help predict future power supply needs. In addition, intelligent network data analytics can help address strategic goals with specific pricing plans tailored to supply, demand, and production models [18].

\section{Smart Transportation}

The use of Big Data in Smart Transport can help improve transport systems by analyzing existing data, providing alternative routes, and minimizing traffic congestion by reducing the number of accidents [19]. The use of smart traffic lights mentioned above is also relevant. 


\section{Smart Governance}

With more information flow from citizens, stronger bridges can be built between citizens and the government. Governments can solve local problems faster and create a citizen-centered governance model that increases transparency between government and the public. This is possible through Big Data [17].

Big Data analytics can play an important role in ensuring smart management. Organizations or institutions with common interests can be easily identified through information analysis that can lead to collaboration between them [15].

\section{Smart Healthcare}

One of the main applications of Big Data is Healthcare.

Big Data relies on different sources such as previous meetings with doctors, social media, and external activities. As a result, it creates a structured image of the customer [22]. The abundance of information available today helps healthcare providers to accurately analyze an individual and accordingly provide appropriate and quality patient care.
The overall goal of Big Data in healthcare is to analyze, identify, and solve medical problems before they represent serious problems. Big Data makes all these procedures efficient.

Big Data also favors telemedicine, one of the key areas of health technology. The rapid growth of this sector, which is expected to reach $\$ 175$ billion by 2026 , is due to emerging technologies such as IoT, Machine Learning, and Artificial Intelligence, along with the widespread use of remote consulting.

\section{Smart Energy Management}

The global energy crisis requires not only the transition from fossil fuels to sustainable renewable alternatives but also effective energy management. With Big Data, everything from individual street lights to energy consumption in individual power grids can be closely analyzed to increase energy distribution efficiency [17].

\section{Smart Security}

Big Data is an ideal technology in terms of security. One of the main goals of Big Data is to ensure the security of large amounts of data. It should be noted that the information 
in the smart city is extreme, the proper management and security of this data can be achieved through Big Data.

In addition, it can provide city internal security. Big Data can allow for a comprehensive sociopolitical survey of areas in focus. At the same time, a protective model can be applied to predict future types of crime [17].

\section{Smart Education}

Smart learning applications will engage people in active learning environments that allow them to adapt to rapid changes in society and the environment. In addition, by relying on Big Data collected in this area and properly processed to obtain the necessary information, we will have a positive impact on the data levels and teaching/learning tools to deliver or obtain the data. In addition, technology can make such opportunities accessible everywhere, including remote or rural areas where it may not be possible to go to school or where people have low incomes and cannot afford other more expensive models [16].

The number of Smart Cities that are constantly evolving is increasing with the technological innovations they use. The top 10 Smart City sequences in the world are as follows: [20]

1. Singapore

2. Dubai

3. Copenhagen

4. Boston

5. Oslo

6. London

7. New York

8. Barcelona

9. Amsterdam

10. Hong Kong

\section{CURRENT SECURITY CHALLENGES} IN SMART CITIES.

Smart cities face many risks as digital and physical infrastructure is crossed. Cities must implement cybersecurity and confidentiality principles at every stage of their development [24].

Since there is a threat of infiltration into the infrastructure of a smart city at any compromised point, the risk increases rapidly, one system can compromise another. In a classic scenario with the weakest channel, one seemingly harmless connected device, being hacked and infected with malicious software, opens up many other devices for penetration, which leads to cascading damage to the entire infrastructure [25] .

An increase in the number of IoT devices in a smart city means more virtual 
attacks can take place. We have also seen from recent DoS attacks based on IoT devices that IoT devices can be captured and used in a Shadownet attack. (Shadownet is an IoT-based botnet that cannot be viewed or tracked with regular browsers or tools.) This method can also prevent users from accessing many services [26]. For example:

- Local service can be stopped. Thus, jobs and services, such as banking, can be stopped because employees cannot go to work.

- Access to drinking water may be interrupted.

- With fake SMS, people can be directed to certain areas, such as a corporate headquarters or government building.

- Fire and theft alarms can be automatically activated across the city.

Cesar Cerrudo revealed that 200,000 road sensors of management from Washington to Melbourne have vulnerabilities that pirates can easily infiltrate. Hackers could take advantage of listed vulnerabilities to control traffic signals as well as modify electronic road signs like speed limits or cause accidents. Hackers, who take advantage of security vulnerabilities in smart systems, such as security systems that can be slightly be intercepted, camera systems that detect a face, can cause major crimes to threaten society. By looking at the listed examples, can draw a conclusion that a system with security vulnerability will be more attractive than a customized system for hackers. However, since all systems are interconnected in smart cities, the hacker infiltrated into any of the systems can cause great turmoil with a domino effect. Therefore, the security strategy should be considered as a whole and at the same time, all security gaps should be minimized or even eliminated for each system [27].

We have summarized the security challenges that smart cities face. Now, let's look at how cybersecurity can be ensured in these systems.

\section{PROVIDING CYBERSECURITY IN SMART CITIES}

Many companies have many problems that can be classified as (a) technology, (b) security, and (c) confidentiality. At the same time, the three areas are interconnected, as insecurity can be called by technological constraints and can bring to a breach of confidentiality. Therefore, it is useful to discuss them together [28]. Security is always called a problem, and smart grid security is the latest in cybersecurity.

Smart cities are equipped with interconnected smart systems so that people can live comfortably, the hackers taking 
over these systems can cause great confusion.

In smart cities, cybersecurity can be provided in two ways: from the inside and out.

In smart cities, security needs to be strengthened from the inside. Some important steps should be taken to strengthen the security architecture in smart cities:

- Use strong encryption.

- Design systems with strong protection to prevent outside interference.

- Provide strong access for control, authentication, and authorization.

- Keep detailed records of the activities.

- Provide separate services for separate subsystems, and then put together the data you want to make available to everyone.

- Create central management, analysis, and control systems through secure and segmented management channels to solve problems.

Set exceptional standards for handling alarms in emergencies, or provide manual intervention.

Strengthening the security of smart cities from the outside: DDoS attacks

Although network segmentation ensures the security, integrity, and continuity of the internal network, we also need to strengthen the internet-facing side of smart cities. DDoS attacks can be easily used to collapse this infrastructure. According to the diameter of the attack and the worst scenario; The city's IT team should develop and implement an effective strategy to prevent DDoS attacks. One of these strategies involves the implementation of solutions where devices are used as overprovisioned, while the other strategy includes hybrid solutions that include devices combined with the cloud [26].

You can manage DDoS attacks that exceed your normal bandwidth thanks to solutions where devices are used as overprovisioned. For example, if your normal user traffic is $1 \mathrm{Gbps}$, you should also have a plan with devices that you can use for a DDoS attack using 20 Gbps bandwidth, and potential bandwidth should be covered by your service provider. If the actual attack is more than the bandwidth offered by your service provider, you may need a hybrid cloud with a cloud-based "scrubber" that can work with your DDoS solution.

As time goes on, smart cities will become smarter by learning something from researchers, from each other, and from events to happen.

\section{CONCLUSION}

In this age of information, both security and the right decision-making process have become important. For this, 
proper data management is very important. In this regard, experts are working on technologies that can solve the problem. One of them is Big Data.

As mentioned above, Big Data has many applications. Many areas of Big Data are covered, from education to health to public administration. Big Data is almost used in many areas where technology is available. And this technology meets expectations. The scope of use of this quartet is constantly expanding.

Big Data is an invaluable tool for proper data management in any field. We also noted the corresponding examples. However, when used in conjunction with IoT, this is a key factor in achieving even greater results than previously thought.

There is a need to create more affordable technological opportunities to further improve future projects such as Smart Cities. Because the information in the future will be many times more than now. In this regard, experts must already work on creating the technology of the future.

Cybersecurity of Smart City is the main challenge that includes addressing slightly technology, application, infrastructure, and information/data security issues. Smart City's cybersecurity is the main challenge it needs international cooperation, it is including experts from around the world. It should be noted that security and confidentiality are key issues and should be addressed.

Finally, Big Data is an important and integral part of Smart Cities. With the help of Big Data, IoT and other technologies, the quality of life of Smart City citizens continues to improve.

\section{REFERENCES}

[1] "Smart applications - the future of applications" - Wipro Limited, Doddakannelli, Sarjapur Road, Bangalore-560 035, India

[2] "How IoT leaders can embrace smart apps" - Johan den Haan, Contributor, InfoWorld | SEP 12, 2018

[3] "Smart Applications: Deliver Insights in Context" - VMWare. https://tanzu.vmware.com/smartapplications

[4] "Internet of Things (IoT) for Automated and Smart Applications" - Yasser Ismail. Published: November 27th 2019. DOI: 10.5772/intechopen.77404

[5] "What is a smart device? - The key concept of the Internet of Things. A conceptualization of the term smart device" - Manuel Silverio. Dec 29, 2019.

https://towardsdatascience.com/wha t-is-a-smart-device-the-key- 
concept-of-the-internet-of-things-

52da69f6f91b

[6] "Internet of Things vs. Smart Home" https://livyprotect.com/en/IoT-vs-smart-home/

[7] "What is the Relationship Between IoT and Big Data?" - Cody Lirette. Published: September 17, 2019

[8] "Smart Apps “ - Mendix. https://www.mendix.com/smartapps/

[9] "Internet of Things and Big Data Analytics for Smart and Connected Communities" - YUNCHUAN SUN, HOUBING SONG, ANTONIO J. JARA, RONGFANG BIE. February 12, 2016.

[10] "Disney: Using Big Data, the IoT and machine learning to enhance the customer experience" Bernard

Marr https://www.bernardmarr.com/def ault.asp? contentID $=1278$

[11] "Big data case study: How UPS is using analytics to improve performance" - Mark Samuels | September 28. 2017 https://www.zdnet.com/article/bigdata-case-study-how-ups-is-usinganalytics-to-improve-performance/

[12] "Ten examples of IoT and big data working well together" - Conner Forrest | March 2. 2015.
[13] "15 EXAMPLES OF IOT AND BIG DATA WORKING IN UNISON" - Mike Thomas. April 10, 2020. https://builtin.com/bigdata/IoT-big-data-analyticsexamples

[14] "Big Data and Smart City" - Dr. Winnie Tang.

[15] "The Role of Big Data in Smart City" - Ibrahim Abaker Targio Hashem, Nor Badrul Anuar, Adewole K. S. International Journal of Information Management · October 2016. DOI: 10.1016/j.ijinfomgt.2016.05.002

[16] "Applications of big data to smart cities" - Eiman Al Nuaimi, Hind Al Neyadi, Nader Mohamed \& Jameela Al-Jaroodi. Journal of Internet Services and Applications volume 6, Article number: 25 (2015)

[17] "5 Applications Of Big Data In Smart Cities" - BW Online Bureau. 27 March, 2019 http://bwsmartcities.businessworld .in/article/5-Applications-Of-BigData-In-Smart-Cities/27-03-2019$168507 /$

[18] "The Role of Big Data Analytics in Smart Grid Management" Bhawna Dhupia, M. Usha Rani, Abdalla Alameen. 11 February 
2020. Part of the Advances in Intelligent Systems and Computing book series (AISC, volume 1054)

[19] "Smart transportation system using big data analytics and broadband telecom network" sanjay gupta, dr. vınodını katıyar. aijrrljsm volume 1, issue 6 (2016,june) （1ssn-2455-6300) online anveshana's international journal of research in engineering and applied sciences

[20] “Top 10 Smart Cities Worldwide: The Future of Urban Evolution" https://en.yeeply.com/blog/top-10smart-cities-worldwide/

[21] "Big Data and Big Data Analytics: Concepts, Types and Technologies" - Youssra Riahi, Sara Riahi. International Journal of Research and Engineering ISSN: 2348-7860 (O) | 2348-7852 (P) Vol. 5 No. 9 | September-October 2018 | PP. 524-528

[22] "Big Data in Healthcare Opportunities and Challenges" Adriana Gabriela ALEXANDRU, Irina Miruna RADU, Mădălina Lavinia BIZON

[23] "How to build a Smart City: with IoT and Big Data" - Olivia Brookhouse. 18 May, 2020 https://business.blogthinkbig.com/

how-to-build-a-smart-city-with-

IoT-and-big-data/

[24] Danda B. Rawat \& Kayhan Zrar Ghafoor "Smart Cities Cybersecurity and Privacy " Pages 303. 2018 December 4

[25] "Cybersecurity challenges for smart cities: Key issues and top threats" August 21, 2019 https://www.helpnetsecurity.com/2 019/08/21/cybersecurity-smartcities/

[26] Derya Aksoy "Akıllı şehirlerde siber saldırıları önlemek" 23 December 2016 https://www.pwc.com/us/en/servic es/consulting/cybersecurity/library /broader-perspectives/smartcities.html

[27] Hakan Kahraman "Akı1lı Şehirlerin Siber Güvenliğii” 22 March https://www.endustri40.com/akillisehirlerin-siber-guvenligi/

[28] Professor Adel S. Elmaghraby "Security and privacy in the smart city" Pages 7. March 2013

[29] Anwaar AlDairi and Lo'ai Tawalbeh "Cyber Security Attacks on Smart Cities and Associated Mobile Technologies". Pages 1086-1091, 2017. 